\title{
Probing the superfluid velocity with a superconducting tip : the Doppler shift effect
}

\author{
A. Kohen ${ }^{1}$, Th. Proslier ${ }^{1}$, T. Cren ${ }^{1}$, Y. Noat ${ }^{1}$, W. Sacks ${ }^{1}$, H. Berger ${ }^{2}$ and D. Roditchev ${ }^{1}$ \\ ${ }^{1}$ Institut des Nanosciences de Paris, I.N.S.P., Universités Paris 6 et 7 , \\ C.N.R.S. (UMR 75 88), 75015 Paris, France and \\ ${ }^{2}$ Institute of Physics of Complex Matter, E.P.F.L., 1015 Lausanne, Switzerland
}

(Dated: September 26, 2018)

\begin{abstract}
We address the question of probing the supercurrents in superconducting (SC) samples on a local scale by performing Scanning Tunneling Spectroscopy (STS) experiments with a SC tip. In this configuration, we show that the tunneling conductance is highly sensitive to the Doppler shift term in the SC quasiparticle spectrum of the sample, thus allowing the local study of the superfluid velocity. Intrinsic screening currents, such as those surrounding the vortex cores in a type II SC in a magnetic field, are directly probed. With $\mathrm{Nb}$ tips, the STS mapping of the vortices, in single crystal $2 H-\mathrm{NbSe}_{2}$, reveals both the vortex cores, on the scale of the SC coherence length $\xi$, and the supercurrents, on the scale of the London penetration length $\lambda$. A subtle interplay between the SC pair potential and the supercurrents at the vortex edge is observed. Our results open interesting prospects for the study of screening currents in any superconductor.
\end{abstract}

A fundamental property of the superconducting (SC) state is its response to an applied magnetic field. In particular, the field penetrates type II superconductors in the form of quantized flux, or vortices, each one carrying a flux quantum of $\phi_{0}=\mathrm{h} / 2 \mathrm{e}$, which are arranged in a lattice [1]. Each vortex is surrounded by screening currents which decay over $\lambda$, the magnetic penetration length, and has a core extending over the coherence length $\xi$. The SC pair potential, $\Delta(\mathbf{r})$, decays from its maximal value outside the core, down to zero in its center. As shown by Bardeen, Cooper \& Schrieffer (BCS), in the spatially homogenous case the SC state has an excitation spectrum given by $E_{\mathbf{k}}=\left(\varepsilon_{\mathbf{k}}^{2}+\Delta^{2}\right)^{1 / 2}$. This results in a unique quasiparticle density of states (DOS) in which a gap of width $\Delta$, with a peak at its edge, opens at the Fermi level. In the vortex state, the DOS in the SC becomes spatially inhomogeneous due to both the currents flowing around the vortices and the variations in $\Delta(\mathbf{r})$. The two different effects are expected to occur on the length scales $\lambda$ and $\xi$ respectively. As can be seen from the BCS spectrum, a consequence of changing $\Delta(\mathbf{r})$ is a modification of the DOS gap width. In addition, as shown by Caroli et al. 2], bound states are formed in the vortex core since $\Delta(\mathbf{r})$ acts as a potential well. The bound states affect the low energy DOS and are significant close to the vortex center.

The Scanning Tunneling Microscope (STM) is an instrument of choice to map the DOS variations on a nanometer scale. The technique is based on the tunneling current $(I)$, flowing between a normal metal tip and a sample, measured as a function of the tip position and the bias voltage $(V)$. Combined with spectro-scopy (STS), the conductance $d I / d V(\mathbf{r}, V)$ reflects the sample local DOS, which has been exploited to study the vortex lattice in several materials 3, 4, 5, 6, 7, 8, 9]. The main focus of these experiments was a detailed study of the vortex core bound states and/or the measurement of $\xi$, as inferred from the spectra due to the spatial variation of $\Delta(\mathbf{r})$. Study of the screening currents, and thus measuring $\lambda$, had proven to be more delicate.
In general when an uniform current flows in a $\mathrm{SC}$ sample, the excitation spectrum can be rewritten as:

$$
E_{\mathbf{k}}=\left(\varepsilon_{\mathbf{k}}^{2}+\Delta^{2}\right)^{1 / 2}+m \mathbf{v}_{\mathbf{F}} \cdot \mathbf{v}_{\mathbf{s}},
$$

where $\mathbf{v}_{\mathbf{F}}$ is the Fermi velocity and $\mathbf{v}_{\mathbf{s}}$ is the superfluid one [10]. As long as the Doppler energy, $m \mathbf{v}_{\mathbf{F}} \cdot \mathbf{v}_{\mathbf{s}}$, is small with respect to $\Delta$, the main effect on the DOS is a reduction in the peak height. However this effect is relatively small, only a few percent in magnitude, and is therefore not effective, with STM, as a means to study the supercurrents. While other methods are sensitive to magnetic field variations, such as Hall probe or SQUID microscopies [1], and are able to measure $\lambda$, they lack the high spatial resolution of STM. Moreover these techniques are insensitive to $\Delta(\mathbf{r})$ and thus cannot be used to determine $\xi$.

In pioneering STS experiments, Hess et al. [4] found that even far from the core the spectra differed from the zero field one, the main difference being a small ingap shoulder. The position of this shoulder is shifted to lower energies as one approaches the core and finally merges with the peaks of the core bound states. They interpreted this effect in terms of the Doppler shift caused by the screening currents. However, as was theoretically shown by [12], this model is not justified when the core states extend to large distances and especially for low energies, when they dominate the spectrum. Thus the the simple model based on the Doppler shift, while giving the correct qualitative result, fails to be quantative and should be replaced by a full solution of the Eilenberger equations [12].

Here we take a different approach and use a superconducting tip, in STM/STS, to directly detect the pair supercurrents, due to their Doppler shift effect on the quasiparticle spectrum. The use of SC tips in low temperature STM was suggested 13] and later realized by several groups 14, 15, 16, 17, 18]. The potential advantages of the SIS (SC-vacuum-SC) configuration are an enhanced spectroscopic energy resolution and the possibility to measure the Josephson pair tunneling [15]. SC tips have so far been applied only at a single point; no scanning spectroscopy has 
yet been reported. A priori, their use to image the vortex lattice could result in complications. First, the local magnetic field might affect the SC properties of the tip and second, there is a force between the diamagnetic SC tip and the vortices. A displacement of the vortices, leading to a distortion of the STS images, is possible. In this letter we report the successful use of superconducting $\mathrm{Nb}$ tips for STS mapping of the vortex lattice in a $\mathrm{NbSe}_{2}$ sample. Owing to the enhanced spectroscopic resolution of the SC tip, we are able to detect both the existence of the in-core bound states, and the supercurrents flowing around the vortex. The latter is achieved by the strong effect of the Doppler shift on the gap-edge peak amplitude.

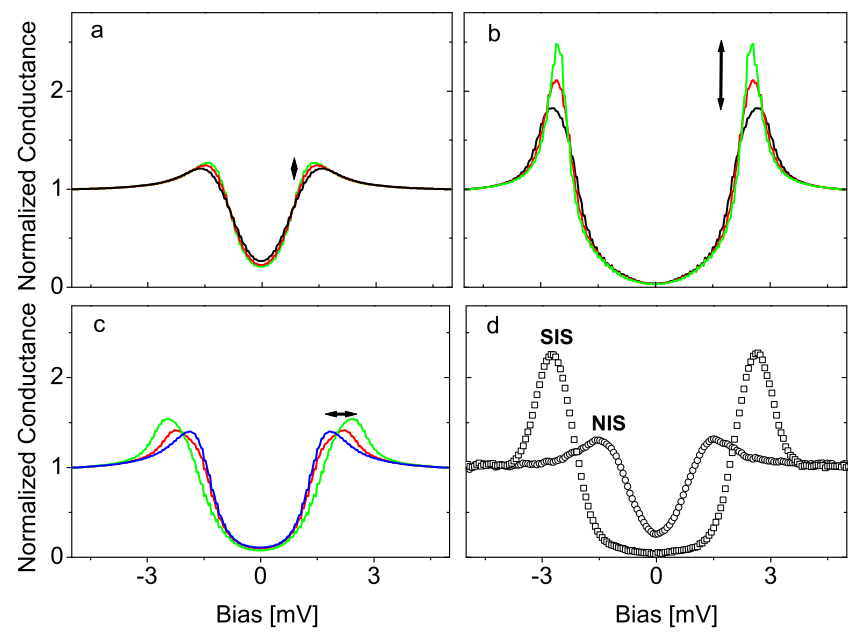

FIG. 1: Calculations of: (a) normalized tunneling conductance for a normal metal tip and a SC sample, with $\Delta$ $=1 \mathrm{meV}, \mathrm{T}=2.3 \mathrm{~K}$, Doppler energy: 0, 0.3, $0.5 \mathrm{meV}$. (b) conductance with a superconducting tip $\Delta_{t i p}=1.5 \mathrm{meV}$, and identical parameters as in (a). (c) SIS conductance with large Doppler shift $=0.65 \mathrm{meV}$ and varying surface gap $\Delta_{\text {sample }}=0,0.3,0.6 \mathrm{meV}$. (d) Experimental tunnel conductance for a $\mathrm{NbSe}_{2}$ sample with a $\mathrm{SC} \mathrm{Nb}$ tip (SIS) and with a normal Pt/Ir tip (NIS), T=2.3 K.

The effect of the supercurrents on a NIS spectrum, as calculated using BCS, is shown in Fig.1a. One can see that if $\mathbf{m}_{\mathbf{F}} \cdot \mathbf{v}_{\mathbf{s}}<\Delta$, where Eq.(1) for the Doppler shift holds, the corresponding change in the tunneling conductance spectrum is very small and thus is difficult to observe experimentally. However, in the SIS geometry (i.e. with a SC tip) the same change in the sample DOS due to the Doppler shift leads to a significant drop in the tunneling conductance peak amplitude, as shown in Fig.1b. The enhancement of the effect is clearly due to the overlap of the tip and sample DOS gap edges. Closer to the vortex core, the principal change of the quasiparticle DOS should be the decrease in the magnitude of $\Delta$, manifested by the gradual shift of the tunneling conductance peaks towards lower energies, see Fig.1c. Thus, in the SIS configuration, one would expect two different behaviors in the SIS tunneling conductance spectra while approaching the vortex core: the decrease in the peak amplitude at large distances and the peak shift to lower ener- gies close to the vortex core. As we will demonstrate, the corresponding length scales may be identified, in a first approximation, as the penetration length $\lambda$ and the coherence length $\xi$, respectively. The peak amplitude variation, together with the Doppler shift energy, give the profile of the supercurrent intensity as a function of distance from the vortex center. By solving the London equation, a quantitative fit yields the values of both $\xi$ and $\lambda$.

$\mathrm{NbSe}_{2}$ crystals, grown using the standard Iodine Vapor Transport technique 19], were studied using our home-made UHV STM setup allowing a minimum temperature of $2 \mathrm{~K}$ and an applied magnetic field up to 6 T. Our low temperature STM unit, described in [20], is digitally controlled and the high-speed data acquisition allows a full spectroscopic mapping of the sample. The preparation and characterization of our Nb tips is reported in [18]. As a simple check, we first measured the spectra of the $\mathrm{NbSe}_{2}$ sample in zero field, and at $\mathrm{T}$ $=2 \mathrm{~K}$ (see Fig.1d). These exhibit the typical features of a SIS junction: sharp peaks appear at the voltages $\pm\left(\Delta_{\text {tip }}+\Delta_{\text {sample }}\right) / e$. For comparison, we show the spectrum using a normal $\mathrm{Pt} / \mathrm{Ir}$ tip (NIS junction). We thus obtain $\Delta_{N b}=1.5 \mathrm{meV}$ and $\Delta_{\mathrm{NbSe}_{2}}=1.0 \mathrm{meV}$, in agreement with their bulk values.

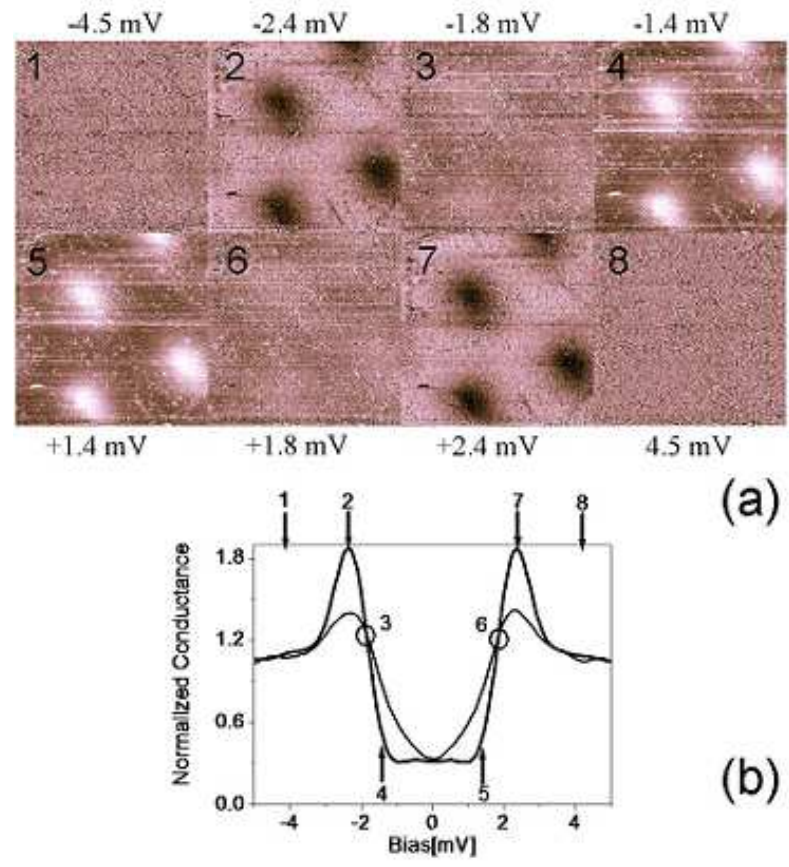

FIG. 2: (a) Fixed scale conductance maps of the vortex lattice, applied field $.06 \mathrm{~T}, 330 \mathrm{~nm} \times 330 \mathrm{~nm}$ area, $\mathrm{T}=4.5 \mathrm{~K}$. The maps $(1-8)$ correspond to the bias voltages as indicated in the figure. The strongest contrast is obtained at $\mathrm{V}= \pm\left(\Delta_{N b}+\Delta_{N b S e_{2}}\right) / e$, maps $2 \& 7$, and at $\mathrm{V}= \pm \Delta_{N b} / e$, maps 4 \& 5 . (b) Normalized conductance spectra measured in the vortex core (NIS) and in between the vortices (SIS). Arrows mark the selected voltages for the conductance maps in (a).

In Fig. 2 we show a typical STS result of a $330 \times 330$ $\mathrm{nm}^{2}$ scan of the sample surface at $\mathrm{T}=4.5 \mathrm{~K}$ and in a magnetic field of $0.06 \mathrm{~T}$. At each point, of a $256 \times 256$ point topographic image, a complete $I(V)$ tunnel- 
ing spectrum was acquired in the sample bias range from $-10 \mathrm{mV}$ to $+10 \mathrm{mV}$. The conductance spectra, $d I / d V(V)$, were directly derived from raw $I(V)$ data. In Fig.2a we present 8 most significant conductance maps selected from 256 measured. The maps are displayed in a fixed gray scale without any additional contrast treatment. It is clear from the maps $\mathrm{n}^{o s} 2$, 4,5 and 7 that the familiar triangular vortex lattice is successfully revealed by STS with a SC tip. Here, the inter-vortex distance of $190 \mathrm{~nm}$ matches the theoretical value $d=\left(2 \phi_{0} / \sqrt{3} B\right)^{1 / 2} \simeq 200 \mathrm{~nm}$, for $B$ $=0.06 \mathrm{~T}$. First, contrary to the case of STS with a non-superconducting tip, the vortices do not appear in the conductance maps near zero bias but rather at higher bias values. The maximum contrast is achieved at $\mathrm{eV} \simeq \Delta_{t i p}$ and $\mathrm{eV} \simeq \Delta_{t i p}+\Delta_{\text {sample. }}$. Second, one observes an almost perfect symmetry of the contrast with respect to the Fermi level. Indeed, the map ${ }^{\circ} 2$ taken at $-2.4 \mathrm{mV}$ is almost identical to $\mathrm{n}^{\circ} 7$ obtained at $+2.4 \mathrm{mV}$. In these two maps the vortices appear black due to the lower tunneling conductance in the vortex cores. The maps $\mathrm{n}^{o s} 4$ and 5 , taken at $-1.4 \mathrm{mV}$ and $+1.4 \mathrm{mV}$ respectively, are also quasi-identical, but the vortices appear in white as the regions of higher conductance.

The origin of the map contrast is better understood from the two spectra plotted in Fig.2b. The first, obtained in the center of the vortex, $\sigma_{N I S}(V)$, shows the characteristic NIS shape, while the second, $\sigma_{S I S}(V)$, taken at a point in between the vortices, shows the SIS one. At voltages above $\left(\Delta_{\text {tip }}+\Delta_{\text {sample }}\right) / \mathrm{e}$, such as $\mathrm{V}_{8}$, both spectra have a common conductance value and no contrast is observed. For lower $V$, a high peak $\left(\right.$ at $\mathrm{V}_{7}$ ) develops in the SIS spectrum at $\mathrm{eV}$ $\simeq \Delta_{\text {tip }}+\Delta_{\text {sample }}$ where it exhibits a higher conductance than the NIS one. The two spectra then cross at $\mathrm{V}_{6}$. For $\mathrm{V}<\mathrm{V}_{6}, \sigma_{S I S}<\sigma_{N I S}$ and a second high contrast can be found for $\mathrm{eV} \simeq \Delta_{t i p}$, at $\mathrm{V}_{5}$. Finally at $\mathrm{V}=0, \sigma_{S I S} \simeq \sigma_{N I S}:$ the contrast is negligible. We see why the contrast is inverted between maps 7 and 5 (respectively 2 and 4), and is small in map 6 (or 3). Qualitatively, features in the sample DOS, besides being enhanced, are shifted in energy by an amount $\Delta_{t i p}$. By selecting the sharpest positive and negative maps, we find the values $\pm 1.4 \mathrm{mV}$ and $\pm 2.4 \mathrm{mV}$, respectively, and thus determine in a different way: $\Delta_{N b}=1.4 \mathrm{meV}$ and $\Delta_{N b S e_{2}}=1.0 \mathrm{meV}$.

To study the vortex bound states and the superfluid velocity profile, we focused on a single vortex lattice unit cell and reduced the temperature to $\mathrm{T}=2.3 \mathrm{~K}$, thus improving both spatial and energy resolutions. The conductance maps at three selected bias voltages (among 256) are shown in Fig.3. The apparent size and shape of the vortices depend sensitively on the particular bias: Map (a), obtained at the SIS spectral peak, $\mathrm{eV} \simeq \Delta_{t i p}+\Delta_{\text {sample }}$, shows a much larger diameter than map (c), obtained at $\mathrm{eV} \simeq \Delta_{t i p}$, which reveals essentially the vortex core. Such an enlargement is precisely due to the Doppler effect of the screening currents. Map (b), obtained at the SIS/SIN crossing- point voltage ( $\mathrm{V}_{6}$ in Fig. $\left.2 \mathrm{~b}\right)$ where little contrast was expected, reveals a particular star shaped halo.

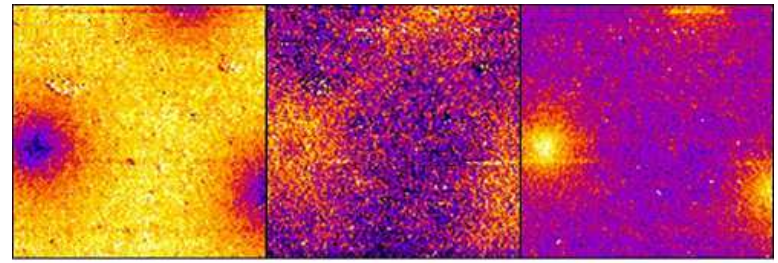

(a)

(b)

(c)

FIG. 3: Conductance maps of a $210 \times 210 \mathrm{~nm}^{2}$ area, $\mathrm{T}=2.3$ $\mathrm{K}$, applied field $.05 \mathrm{~T}$. Maps selected at voltages: $V=2.6$ $\mathrm{mV}, 2.2 \mathrm{mV}$ and $1.6 \mathrm{mV}$ corresponding to (a) the SIS peak, (b) the SIS/SIN intersection point and (c) the NIS peak. These emphasize respectively: (a) the long range variations in the SIS peak height, (b) the vortex star shaped halo and (c) the vortex core. The scale of each map is readjusted to obtain the best contrast, hence the apparent lower resolution in (b).

The dynamics of the spectral shape, for different distances from the vortex center, is displayed in Fig.4. To reduce noise, each spectrum is the average over a circle of radius $r$, concentric with the vortex. One clearly sees the evolution from a SIS spectrum (A) obtained far from the vortex, with a wide apparent gap and pronounced peaks, to NIS spectra with low peaks and a narrower gap, obtained in the vicinity of the core. At even smaller distances $(r \lesssim 10 \mathrm{~nm})$ we observe a subtle dip - hump feature $(\mathrm{D}, \mathrm{H})$ developing just above the gap edge, becoming more pronounced as we approach the vortex center (B). There, a slight increase of the amplitude of the peak is observed. The effect is the signature of the vortex core states which exist near the Fermi energy, but which are shifted to a voltage above $\Delta_{t i p} /$ e in our case.

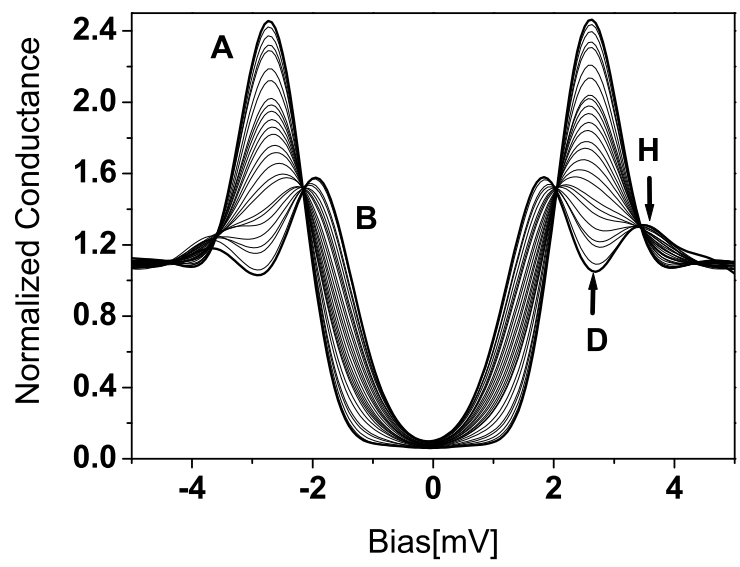

FIG. 4: Evolution of the conductance spectra as a function of distance from the vortex center. Far from the vortex (A) the spectra show SIS features with high peaks at $\left(\Delta_{\text {tip }}+\Delta_{\text {sample }}\right) / e$. The peak height is first lowered and then followed by a shift to a lower voltages, near $\simeq \Delta_{t i p} / e$. Close to the vortex center (B) a dip hump feature appears $(\mathrm{D}, \mathrm{H})$, associated with a slight increase in the peak. 
The spatial changes in the spectra, as a function of distance $r$ from the core, are summarized in Fig. 5 by the plots of the peak position and amplitude. Starting from a large distance, the peak height, initially at 2.4 , is continuously diminished, finally leveling off at a value $\sim 1.5$ at $r=r_{c} \simeq 110 \AA$ (dashed line). This lowering of the peak amplitude (predicted in Fig.1b and clearly visible in Fig. 4) is due to the increase in the superfluid velocity as one approaches the vortex core, which saturates at the critical velocity, $\mathbf{v} \simeq \mathbf{v}_{c}$ at $r \simeq r_{c}$. On the contrary, the peak energy is roughly constant (at $\sim 2.6 \mathrm{meV}$ ) for all $r \gtrsim 2 r_{c}$ but then decreases rapidly $\left(r \sim r_{c}\right)$ down to its minimum value $\sim 1.8 \mathrm{meV}$ at the vortex center. Thus, the peak position as a function of $r$, shifted downwards by $\sim \Delta_{t i p}$, matches the pair potential profile. As the mid-point of this profile is commonly used to estimate $\sqrt{2} \xi$, we find directly $\xi \simeq 80 \AA$ and from the peak amplitude profile, $\lambda \simeq 750 \AA$. In short, Fig. 5 gives a picture of the pair potential and supercurrent profiles of a vortex, of core radius $r_{c}$.

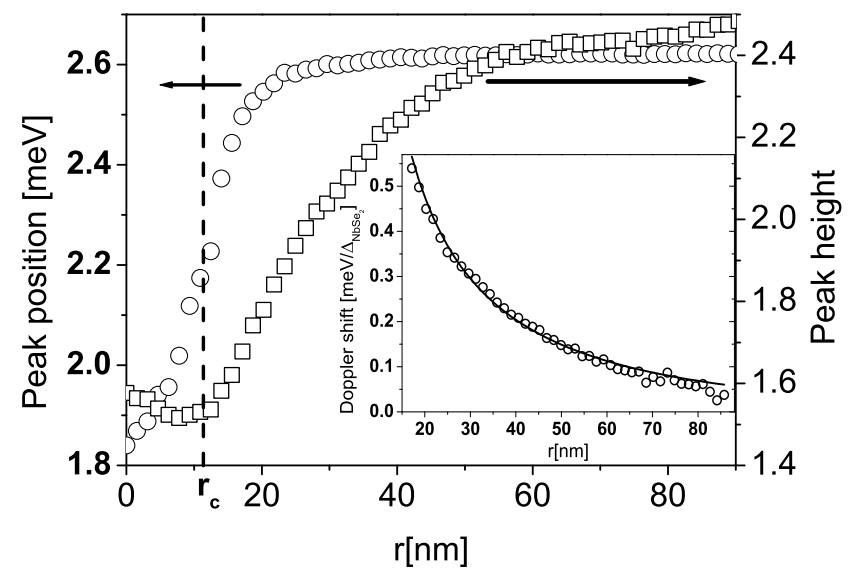

FIG. 5: Gap-edge peak energy (circles) and amplitude (squares) as a function of distance from the vortex center. Inset shows the Doppler shift energy, in units of $\Delta_{\mathrm{NbSe}_{2}}$, as a function of distance from the vortex core (circles). The solid line is a theoretical fit, $\frac{\pi}{2} \frac{\xi}{\lambda} \mathrm{K}_{1}(r / \lambda)$ with the best fit obtained using $\lambda=68 \mathrm{~nm}$ and $\stackrel{\xi}{=}=6.6 \mathrm{~nm}$.

The values of $\lambda$ and $\xi$ may be extracted in a different way, through the Doppler shift energy (from Eq. 1 ) as a function of $r$ (see inset of Fig.5). We have fitted the data with the function: $\frac{\pi}{2} \frac{\xi}{\lambda} \mathrm{K}_{1}(r / \lambda)$, derived from the London equation for an isolated vortex, where $K_{1}(x)$ is the modified Bessel function of order 1. (This approximation neglects Fermi surface and gap anisotropies.) The best fit is found using $\xi=66 \AA$ and $\lambda=680 \AA$ and is shown as the line in the inset. Finally, the Doppler shift at $r=\xi$, together with the Fermi momentum $m v_{F}=\hbar k_{F} \simeq \hbar \pi / 2 a$, where $a \simeq 3.5 \AA$ is the lattice constant, leads to $v_{c} \approx 180 \mathrm{~m} \mathrm{~s}^{-1}$ for the magnitude of the critical velocity.

Our results, i.e. the vortex mapping using a SC tip, the detailed evolution of the SIS to SIN spectra, and the Doppler shift energy variation with distance from the core, all indicate that no perturbation was observed, arising from an interaction between the SC diamagnetic tip and the vortices. Furthermore, the $\mathrm{Nb}$ tips remain superconducting even under a field up to $\sim 0.3 \mathrm{~T}$ (the field in the vortex core for an applied field of $0.05 \mathrm{~T}$ ) a higher value than the bulk critical field, $0.2 \mathrm{~T}$. Our previous studies on the effect of magnetic fields on our $\mathrm{Nb}$ tips, with a normal metal $\mathrm{Au}$ sample, have shown that the tip critical field is enhanced up to $1 \mathrm{~T}$ (at $\mathrm{T}=4.2 \mathrm{~K}$ ). This result is due to the tip's apex size being smaller than both $\xi$ and $\lambda$ with the critical field depending on the exact tip geometry 18. In a small number of experiments, currently under study, we did observe a distorted vortex shape.

In conclusion, we have presented detailed STS mapping of the vortex lattice using a superconducting tip, which opens new possibilities for studying the velocity profile of the pair currents, in any SC sample (type I included). We have demonstrated that the features in the sample DOS are shifted in energy by an amount $\Delta_{t i p}$, as expected, but the principle result is the direct effect of the Doppler shift on the SIS peak amplitude, allowing a detailed mapping, on the nanometer scale, of the currents in the superconductor. It could then be applied to cases where there is an external current source, an oblique magnetic field, or to confined superconductors, where giant vortices are predicted. The presence of spontaneous local currents in highTc superconductors, near the low-temperature pseudogap transition, could be checked. Finally, we have demonstrated the stability of our $\mathrm{Nb}$ tips for use in STS, paving the way for the future measurement of the Josephson current.

The authors thank F. Debontridder and F. Breton for their technical assistance. Sample preparation was supported by the NCCR research pool MaNEP of the Swiss NSF.

[1] A. A. Abrikosov Soviet Physics JETP 5, 1174 (1957)

[2] C. Caroli et al. Phys. Lett. 9, 307 (1964).

[3] H. F. Hess et al. Phys. Rev. Lett. 62, 214 (1989)

[4] H. F. Hess et al. Phys. Rev. Lett. 64, 2711 (1990)

[5] Ch. Renner et al. Phys. Rev. Lett. 67, 1650 (1991)

[6] Y. De Wilde et al. Phys. Rev. Lett. 78, 4273 (1997)

[7] H. Sakata et al. Phys. Rev. Lett. 84, 1583 (2000)

[8] S.H. Pan et al. Phys. Rev. Lett. 85, 1536 (2000)

[9] M. R. Eskildsen et al. Phys. Rev. Lett. 89, 187003 (2002)

[10] P. Fulde Tunneling Phenomena is Solids, Plenum Press New York 1969, 427

[11] For a thorough review see: P. Björsson, Ph.D. Thesis, Stanford University, 2005, and refs. therein.

[12] T. Dahm et al. PRB 66144515 (2002)

[13] R. Meservey Phys. Scr. 38, 272 (1988)

[14] S. H. Pan, et al., Appl. Phys. Lett. 73, 2992 (1998)

[15] O. Naaman, et al., Rev. Sci. Instrum. 72, 1688 (2001)

[16] H. Suderow, et al., Physica C 369, 106, (2002)

[17] F. Giubileo et al. Phys. Rev Lett. 87, 177008 (2001)

[18] A. Kohen et al. Physica C 49, 18 (2005)

[19] R. Bel, et al., Phys. Rev Lett. 91, 66602 (2003)

[20] T. Cren et al. Europhys. Lett., 54 (1), 84 (2001) 